\title{
Virtual environment pedestrian training programs for children: a review of the literature
}

\author{
Shantelle Novak
}

The following manuscript was prepared for under the supervision of Professor Barbara Morrongiello, Department of Psychology, College of Social and Applied Human Sciences

\begin{abstract}
Pedestrian injuries are among the most frequent and serious causes of injury to children. Exploration of an appropriate and effective training program is needed. Many children's pedestrian training programs have been implemented in the past in attempts to alleviate this problem, but they have been unsuccessful in transferring the knowledge the children learned to actual behaviours exhibited. Literature suggests developing a program where children can not only learn about safe pedestrian behaviours, but be given the opportunity to discuss possible actions and outcomes with their peers under guidance of an adult. To help children in the generalization of knowledge to behaviour, training programs should also give children the chance to practice newly learned skills in a simulated environment using computer-based technology. Virtual environment training programs have been successful in the past, especially computer games, which are both educational and entertaining and thus appealing to children. According to literature, development of a virtual environment pedestrian training program appropriate for the child's current cognitive abilities will allow them to practice safe pedestrian practices. The skills learned from such a program can directly transfer to behaviours in real-life situations, and would contribute to decreasing the prevalence of childhood pedestrian injuries.
\end{abstract}

$\mathrm{C}$ hildren unnecessarily suffer from pedestrian injuries at a level much higher and much more severe than that of adults. $^{9}$ In an attempt to reduce these high injury levels, many educational injury-prevention and road safety programs have been implemented in school and home settings, unfortunately, without long-term success. The purpose of this paper is to outline past research in the realm of child pedestrian skills, and to explore the potential benefits of using virtual environments to improve children's pedestrian behaviours. Virtual environments are computersimulations designed to mimic real-world situations by providing visual, auditory, and sometimes tactile information to the people submersed in the environment. This paper analyzes how virtual environments such as computer games can not only increase road safety knowledge among children, but can also be successful in transferring that knowledge to behaviour in real-world situations. Successful child pedestrian training programs in both real-life and virtual environments will be discussed. Factors to consider when designing a pedestrian training program for children will be outlined, including the transfer of knowledge from training to actual behaviours in real situations, the role of adult guidance and peer collaboration on learning, and the effect of cognitive abilities such as attention. Finally, implications of past studies will be discussed along with suggestions for future research.

\section{Past SUCCeSful ReAl-Life TRAINING PROGRAMS}

Due to the alarmingly high levels of child pedestrian injuries, many programs aiming to promote pedestrian safety skills in children have been implemented. While many of these programs have been successful in increasing children's knowledge and awareness of road safety, few have been able to transfer the children's newly-acquired skills to application in the real world. The few that were able to transfer knowledge to behaviour typically were practical training programs where children actively made judgments about traffic events and received feedback from a supervisor on the accuracy of their judgments. These practical training programs have generally taken place in the real world, where children go outdoors and stand alongside the road, however, several programs are still conducted indoors using a computer-simulate environment. Practical training programs in both real and simulated environments have had success in improving pedestrian skills such as children's roadside crossing judgments, ${ }^{16}$ ability to cross at parked cars and intersections, ${ }^{10}, 11,17$ using designated crossings, ${ }^{18,} 19$ identifying roadside dangers, $2,15,17,20$ and increasing children's awareness of others' intentions. ${ }^{18}$ While this appears to be an effective way to train children on pedestrian skills, practical training can be time-consuming and labour intensive. Real traffic environments - as 
appropriate as they may be - are unpredictable and therefore make it difficult for the researcher to control and test for all the variables and to design a program that ensures a variety of traffic situations. Simulation training, through virtual environments and computer games, seems to provide a solution to these problems.

\section{USAGE OF VIRTUAL ENVIRONMENTS TO TRAIN BEHAVIOURS}

To overcome the barriers involved in practical real-world training, computer games and virtual simulation training programs have been designed. Training programs that use computer simulations are advantageous since they provide the opportunity to create a scenario that is realistic enough for children to learn the skills necessary to survive in a realworld situation. Virtual programs also allow researchers the ability to control for all variables and to reproduce identical traffic situations for each participant, both of which are expensive and nearly impossible to do in real life. ${ }^{7,} 12$ Along with many other researchers, Tolmie et al. has proven that successful training does not necessarily need to take place at the roadside. ${ }^{20}$ Not only does computer-based training produce a desired change in behaviour, it may also be more effective and more efficient than roadside training. ${ }^{20}$ By using computer-simulated programs, children have been trained to promptly cross the road and miss fewer safe opportunities to cross, ${ }^{16}$ find safe places to cross the road, ${ }^{18}$ look "left-right-left", and stop at the curb before crossing the road. ${ }^{7}$ Successful virtual environment training programs will be discussed, along with the outcomes of studies that utilized computer games and videos to train behaviour.

\section{Successful Virtual Environment Studies}

The effectiveness of teaching pedestrian safety to children using virtual environments has been explored in the past. ${ }^{7}$ McComas et al. explored whether training in a virtual environment can improve children's skills and knowledge of pedestrian safety, and if that learning was transferrable to behaviour in the real world. ${ }^{7}$ Children in grades 4-6 (approximately 9-11 years old) from both suburban and urban schools engaged in a desktop virtual simulation program designed to both educate and train them on road safety skills, specifically, on how to cross an intersection safely. Four behaviours of the children were measured: (1) walking on the sidewalk versus walking on the street (2) stopping at the curb (3) looking "left-right-left" before crossing and (4) staying attentive while crossing the street.

The results of the children who participated in the training were compared to a control group of children who received an unrelated virtual program. The researchers hypothesized that children who were trained in the virtual environment would have significantly better pedestrian safety scores after training than before. They also hypothesized that trained children would have significantly higher real-world pedestrian safety scores than the control group of children who did not receive the training. Results show that, to some extent, both hypotheses were confirmed. There was a significant difference in street crossing behaviour of all children, indicating there was a learning effect of pedestrian safety rules. However, only children in the suburban school were able to transfer their safety knowledge to the real world. The researchers found that this was because most children in the urban school had English as their second language. For future studies, McComas et al. recommended that a virtual environment program (that is as similar to the real-life situation as possible) be designed, and that social factors such as peer pressure be built into the virtual environment. ${ }^{7}$

A computer-simulated traffic environment was used by Tolmie et al. to examine the potential of computer-based training on four pedestrian skills: (1) safe place finding, (2) roadside search, (3) gap timing and (4) perception of others' intentions. ${ }^{18}$ They tested children 5-10 years old in groups of three guided by an adult trainer. The adult encouraged two forms of social interaction: adult guidance, where the adult offered suggestions; and peer collaboration, where the children discussed possible solutions among themselves. The study was conducted during four 30 minute training sessions using a computer-simulated game where the children were asked to decide the location and the time when it was safe for the computer character to cross the road. The findings were uniformly positive as there were significant improvements in roadside behaviour in all four pedestrian skills, as compared to a control group of children who did not receive any training. There were clear benefits across all ages, with the most improvements made by the 8 and 10 year olds, and the least improvement made by the 6 year olds. Tolmie et al. confirmed the potential of computer-based training, but suggests it be used in addition to, not substituted for, roadside training. ${ }^{18}$

Due to the success of Tolmie et al., ${ }^{18}$ Thomson et al. aimed to build on the findings by conducting a similar study that examined the roadside crossing judgments of children ages 7, 9, and 11 years old. ${ }^{16}$ There were four research questions involved in the study: (1) Are children's pedestrian judgments amenable to training? (2) If so, are they equally amenable to training? (3) What are the conceptual and strategic issues involved in learning to cross through traffic gaps? (4) What is the extent to which improved conceptual and strategic thinking assists in generalizing to children's behavioural judgments at the roadside? The answers to these questions were explored in a similar manner to Tolmie et al. ${ }^{18}$ by immersing a group of children in a computersimulated traffic environment and having an adult volunteer ask them to identify points that were safe for the virtual character to cross the road. The children were tested before and after training and were compared to a control group of children who were tested at the same times, but did not participate in the training. The researchers hypothesized that 
training would lead to improvements in children's conceptual understanding of the crossing task, and that these improvements would generalize to children's behaviour at the roadside.

These hypotheses were confirmed. Children's conceptual scores improved, as well as four of the six behavioural measures. Thomson et al. found that trained children crossed the street more promptly by taking less time to start crossing and by crossing more quickly. ${ }^{16}$ When children estimated their crossing times, trained children's estimations were found to be more closely aligned with their actual crossing times than untrained children. Trained children also missed fewer safe crossing opportunities because they were not as hesitant to cross when there was a smaller gap in traffic. This study proved to be quite successful, especially since all age groups improved to the same extent and, when tested 8 months later, there was no deterioration of skills learned. The researchers attributed most of this success to the reflection and discussion activities. In this conceptual portion of the training, children were encouraged to make explicit decisions, link situations, and discuss points among their peers. ${ }^{16}$ Future child pedestrian training programs may benefit by adding a conceptual piece to the training since it has been demonstrated to increase the longevity and generalization of learning.

\section{Usage of Computer Games}

Some virtual environment programs have been designed as computer "games", thus serving both educational and entertainment purposes. Pedestrian safety concepts have been introduced in an innovative way through use of computer games. ${ }^{7}$ One successful study using a computer game is looked at in depth to provide some background on the effectiveness of these educational entertainment tools.

Computer games have been used to increase the knowledge of fire and street safety in children ages 4-10 years old diagnosed with fetal alcohol syndrome. ${ }^{5}$ It was hypothesized that after successfully mastering the computer game, the children would demonstrate a significant improvement in their ability to verbalize safety rules, and then later be able to generalize these rules to behaviours in the real world. They also hypothesized that the learning would be long-term, and that no significant change in their scores would be found when tested one week later. Results showed that children's knowledge on fire and street safety was significantly improved, and $72 \%$ of the children were able to transfer knowledge learned in the game to a behavioural setting. According to findings, this study proves to be a very effective method for teaching safety skills to alcohol-affected children.

Coles et al. directed attention to the usefulness and effectiveness of computer games in training children. ${ }^{5}$ Most children enjoy playing computer games and will spend hours on end doing so with minimal supervision. He therefore concluded that it would be in advantageous to implement learning into games. Computer games provide the opportunity for children to learn at their own rate, repeating stages of the training as many times as required to achieve mastery of the skill..$^{5}$

\section{Usage of Educational Videos}

In an attempt to teach children about road safety, another method of entertainment was used in Britain, however, it was unsuccessful. Copies of a road safety video released by a popular children's entertainment group were distributed to 120 families who had 5-year-old children. ${ }^{22}$ The families were then instructed to watch the video at home and record how many times the video was viewed daily. A study was designed to measure if viewing the video had any effect on either the children's knowledge about road safety or the parents' attitudes towards children's road safety. ${ }^{22}$ They found the video, when used in this way, had no educational impact on either the parents or the children. A limitation of this study was that it only measured knowledge and awareness, not actual behaviour. Further research should be done to investigate the potential benefits of viewing this video before investing in a video-watching intervention program.

Evidently, virtual environment programs can be successful in training children's behaviours. Programs that use virtual environments provide a safe, controlled environment in which children can be taught pedestrian skills. They also give the opportunity for consistent feedback, practice, and repetition, which are necessary in order to successfully transfer skills to behaviour. Given the current research, it would be ineffective to design a training program using a video; rather, results suggest that it would be beneficial to use a computer game. In addition to being educational, computer games are also entertaining and make learning more enjoyable and "fun" for kids. Computer games are also very accessible and can be distributed quickly and easily to both schools and homes. ${ }^{7}$

\section{FACTORS TO CONSIDER WHEN DESIGNING A VIRTUAL ENVIRONMENT PEDESTRIAL TRAINING PROGRAM}

There are many factors to consider when designing a virtual environment training program to improve children's pedestrian skills. Current research gives insight as to which factors are most important. These factors include the ability to generalize knowledge to behaviours in the real world, the effect of adult guidance and peer collaboration on learning, ${ }^{20}$ and the role of cognitive abilities such as attention on children's road behaviour., $3,4,13,14$ 


\section{Generalization of Knowledge to Behaviour}

What a person knows is typically quite different than what a person does. As an example, the general public is aware and knows the implications of drinking and driving, but nevertheless, people still drive after consuming alcohol. In the same fashion, most children have been taught road safety rules either by their parents or teachers, but the prevalence of child pedestrian injuries remains quite high. Simply knowing road safety rules is generally unrelated to road crossing performance. $^{20,21}$ Verbal instruction of road safety rules, traditionally given in the classroom, are largely ineffective in providing children with skills that will generalize to realworld situations. ${ }^{1}$ Instead of telling children to "be careful" and "look before crossing the street", educators should emphasize appropriate street-crossing behaviour. Pedestrian competence, the ability to generalize to real road-crossing situations, is characterized by skill rather than knowledge and is dependent upon the development of psychological processes needed for skilled pedestrian behaviour. ${ }^{16}$ In past studies, the focus has been on assessing knowledge and attitudes since they are much easier to measure than actual behaviours. ${ }^{22}$ Studies that assess behaviours should therefore be emulated. ${ }^{5,16,18}$ To design an educational program that is more likely to successfully reduce the incidence of child pedestrian injuries, future studies should aim to assess whether the knowledge learned in training will transfer to actual behaviours in the real world.

\section{The Role of Adult Guidance and Peer Collaboration}

Because of success in past studies, ${ }^{16,}{ }^{18}$ Tolmie et al. examined the impact of adult guidance and peer collaboration on simulation-based training in roadside search skills of children 5-10 years old. ${ }^{20}$ The first part of the study looked at the impact of adult guidance versus peer discussion on children's roadside search skills. The participants were separated into three groups. One group of children worked through the training program one-on-one with the adult, who offered guidance and advice to the child. A second group of children collaborated amongst themselves in groups of three. The third group of children did not receive any training and served as the control group. Results showed that children who were guided by an adult improved significantly more than the children in the peer discussion group. To examine this further, the second part of the study compared two groups of children, one guided by an adult and the other not.

The researchers found that children improved even more when adult scaffolding was supplemented by peer discussion. They attributed this finding to a combination of the adult dialogue and the children's exploration of conflicting ideas. The implications of this study show how important it is for a program to include a dimension that generates deeper understanding of the material, such as social interaction. Without this social piece, attempts to promote pedestrian skills through simple modelling of appropriate behaviour would likely be less effective. ${ }^{8}$ This study suggests that future studies should include a form of social interaction such as adult scaffolding and peer discussion in the training program.

\section{The Effect of Cognitive Abilities on Roadside Behaviour}

The development of children's cognitive abilities need to be taken into consideration when developing a pedestrian training program, since they have an effect on whether or not the child will be able to learn effectively from the program. The development of concentration and various forms of attention are the most researched forms of cognitive skills in children, and are thus discussed below.

Dunbar et al. studied two cognitive skills in children ages 4-10 years old: concentration and the ability to switch attention. ${ }^{6}$ Switching attention was measured through use of a computer game that required the child to switch their attention between two activities. Concentration was measured by use of distractors while the children were playing the game. The researchers hypothesized sources of risk to be when the child failed to ignore potentially distracting events or failed to switch attention to the road crossing task. Results showed that children who were better at switching attention were more likely to show awareness of traffic when crossing the road. They also found that children who maintained concentration when challenged by a distracting event crossed the road in a less reckless manner.

These results confirm the hypothesis, since children who are lacking in both concentration and the ability to switch attention would be less aware of traffic and would cross the street in a reckless manner, posing as a potential risk to injury. Dunbar et al. found a significant age trend in the results, with the older children switching attention faster and being less susceptible to distraction. ${ }^{6}$ They found the 4 year olds to have significantly lower attention switching abilities and weaker concentration skills than the other children. Interestingly, these results were also related to road-crossing behaviour; children who were weaker at these tasks were also found to be less skilled at dealing with the road tasks. This has tremendous implications, suggesting that young children may be at an even greater risk when crossing the road. Future research should be conducted to discover a way to improve skill development in attention switching and concentration, and program designers should keep in mind the development of these skills.

Tabibi and Pfeffer also looked at the role of attention in children's road safety. ${ }^{14}$ Specifically, they examined the effect of distractors and the role of attention in identifying safe and dangerous road-crossing sites. The participants in their study were separated in four age groups: 6-7 years old, 8-9 years old, 10-11 years old and adults (mean age 23.3 years old). They measured this by presenting pictures on a computer either with or without visual and auditory distractions. Results showed that distractors and low levels of attention have a negative effect on children's ability to identify safe and dangerous road-crossing sites. They found 
an age effect, with the youngest group (6-7 year olds) scoring significantly lower on all attention tests. ${ }^{14}$ The ability to identify safe and dangerous road-crossing sites as well as performance on the attention tests were also found to improve with increasing age. The percentage of correct responses increased with age up to 10-11 years, but there was no significant difference between the 10-11 year olds and the adult group. In addition, the time taken to identify safe and unsafe road-crossing sites decreased with age. With regards to time, it took longer to identify the sites when distractors were present. Gender effects were not found. Further research should take into account the development of attention and concentration in children. ${ }^{6,13,14}$

\section{IMPLICATIONS FOR FURTHER RESEARCH}

As can be noted above, there have been a number of studies conducted within the scope of training child pedestrian skills using virtual environments. It is imperative that any further research in this field takes into consideration past findings and suggestions. The most evident suggestion is that programs need to be designed in such a way that the knowledge learned in training be generalized and transferred to behaviours in real-life situations. This can be ensured by developing a conceptual piece to the program where, for example, social interaction such as adult guidance and peer collaboration are part of the program design. ${ }^{16,18,20}$ Aside from the generalization of behaviours, many other factors should be taken into consideration when designing future studies. McComas et al. recommended that a training program be designed to include social factors such as peer pressure, in order to make training as similar to real-life situation as possible. ${ }^{7}$ The development of cognitive skills, such as attention and concentration were also suggested to take into consideration. ${ }^{6}{ }^{14}$ Future research that utilizes these suggestions is recommended and will likely have positive outcomes.

\section{CONCLUSiON}

While there are many areas left to study in more depth, the future looks bright for virtual environment behaviour training programs. The potential of computer-based training has been confirmed, ${ }^{7,} 16,18$ including computer games, ${ }^{5}$ but excluding videos. ${ }^{22}$ Literature suggests this type of training program would likely work when applied to children's pedestrian injury prevention programs. However, computer-based training is suggested to be used as a complement to roadside training and traditional verbal instruction, not as a replacement. ${ }^{18}$ There have been many successful studies conducted in the past, and future studies should likely be successful as well if the aforementioned factors are implemented and utilized in the program. It is hopeful that if a successful child pedestrian virtual environment training program was developed and properly executed, the rates of child pedestrian injuries will decrease.

\section{REFERENCES}

1. Ampofo-Boateng, K., \& Thomson, J.A. (1989). Child pedestrian accidents: a case for preventative medicine. Health Education Research, 5, 265-274.

2. Ampofo-Boateng, K., Thomson, J. A., Grieve, R., Pitcairn, T., Lee, D. N., \& Demetre, J. D. (1991). A developmental and training study of children's ability to find safe routes to cross the road. British Journal of Developmental Psychology, $11,31-45$.

3. Barton, B. (2006). Integrating selective attention into developmental pedestrian safety research. Canadian Psychology, 47(3), 203-210.

4. Clancy, T. A., Rucklidge, J. J., \& Owen, D. (2006). RoadCrossing Safety in Virtual Reality: A Comparison of Adolescents With and Without ADHD. Journal of Clinical Child and Adolescent Psychology, 35(2), 203-215.

5. Coles, C. D., Strickland, D. C., Padgett, L., \& Bellmoff, L. (2007). Games that "work": Using computer games to teach alcohol-affected children about fire and street safety. Research in Developmental Disabilities, 28(5), 518-530.

6. Dunbar, G., Hill, R., \& Lewis, V. (2001). Children's attentional skills and road behaviour. Journal of Experimental Psychology: Applied, 7(3), 227-234.

7. McComas, J., MacKay, M., \& Picik, J. (2002). Effectiveness of virtual reality for teaching pedestrian safety. CyberPsychology \& Behavior, 5(3), 185-190.

8. Rivara, F. P., Booth, C. L., Bergman, A. B., Rogers, L.W., \& Weiss, J. (1991). Prevention of pedestrian injuries to children: Effectiveness of a school training program. Pediatrics, 88, 770-775.

9. Rodriguez, J. G., \& Brown, S. T. (1990). Childhood injuries in the United States. American Journal of Disease Control, 144, 627-646.

10. Rothengatter, J. A. (1981). Traffic Safety Education for Young Children. Swets \& Zeitlinger, Lisse.

11. Rothengatter, J. A. (1984). A behavioural approach to improving traffic behaviour of young children. Ergonomics, 2, 147-160.

12. Seward, A. E., Ashmead, D. H., \& Bodenheimer, B. (2007). Using virtual environments to assess time-to-contact judgments from pedestrian viewpoints. ACM Transactions on Applied Perception, 4(3), 1-19.

13. Tabibi, Z. \& Pfeffer, K. (2003). Choosing a safe place to cross the road: The relationships between attention and identification of safe and dangerous road-crossing sites. Child Care, Health \& Development, 29(4), 237-244.

14. Tabibi, Z. \& Pfeffer, K. (2007). Finding a safe place to cross the road: The effect of distractors and the role of attention in children's identification of safe and dangerous road-crossing sites. Infant and Child Development, 16, 193206. 
15. Thomson, J. A., Ampofo-Boateng, K., Pitcairn, T., Grieve, R., Lee, D. N., \& Demetre, J. D. (1992). Behavioural group training of children to find safe routes to cross the road. British Journal of Educational Psychology, 62, 173183.

16. Thomson, J. A., Tolmie, A. K., Foot, H. C., Whelan, K. M., Sarvary. P., \& Morrison, S. (2005). Influence of Virtual Reality Training on the Roadside Crossing Judgments of Child Pedestrians. Journal of Experimental Psychology: Applied, 11(3), 175-186.

17. Thomson, J. A., \& Whelan, K. M. (1997). A community approach to road safety education using practical training methods: The Drumchapel project. Road Safety Research Rep. No. 3. London: Department of the Environment, Transport \& the Regions.

18. Tolmie, A., Thomson, J., \& Foot, H. (2002). Computerbased support for the training of children's pedestrian skills: Software design and evaluation of impact. Computers in
Education, 1, 515-519.

19. Tolmie, A. K., Thomson, J. A., Foot, H. C., Burkes, M., Wu, C., Towner, E. L. M., et al. (2003). Training children in the safe use of designated crossings. Road Safety Research Rep. No. 34. London: Department for Transport.

20. Tolmie, A., Thomson, J. A., Foot, H. C., Whelan, K., Morrison, S., \& McLaren, B. (2005). The effects of adult guidance and peer discussion on the development of children's representations: Evidence from the training of pedestrian skills. The British Journal of Psychology, 96, 181204.

21. Zeedyk, M. S., Wallace, L., Carcary, B., Jones, K., \& Larter, K. (2001). Children and road safety: Increasing knowledge does not improve behaviour. British Journal of Educational Psychology, 71, 573-594.

22. Zeedyk, M.S. \& Wallace, L. (2003). Tackling children"s road safety through edutainment: An evaluation of effectiveness. Health Education Research, 18(4), 493-505. 